\title{
Impulsive Fractional Semilinear Integrodifferential Equations with Nonlocal Conditions
}

\author{
Xue Wang $(\mathbb{D}$ and Bo Zhu \\ School of Mathematics and Quantitative Economics, Shandong University of Finance and Economics, Jinan 250014, China
}

Correspondence should be addressed to Xue Wang; 20058509@sdufe.edu.cn

Received 28 April 2021; Accepted 11 June 2021; Published 21 June 2021

Academic Editor: Xinguang Zhang

Copyright (c) 2021 Xue Wang and Bo Zhu. This is an open access article distributed under the Creative Commons Attribution License, which permits unrestricted use, distribution, and reproduction in any medium, provided the original work is properly cited.

This paper is devoted to a class of impulsive fractional semilinear integrodifferential equations with nonlocal initial conditions. Based on the semigroup theory and some fixed point theorems, the existence theory of PC-mild solutions is established under the condition of compact resolvent operator. Furthermore, the uniqueness of PC-mild solutions is proved in the case of the noncompact resolvent operator.

\section{Introduction}

The fractional evolution equation has been applied to many fields, and scholars have obtained abundant research achievements [1-13]. Impulsive fractional integrodifferential equations can describe some phenomena which often occur in physics, geology, and economics, for instance, earthquake, the closing of the switch in the circuit, and so on. Many scholars are committed to this subject and have achieved plentiful results [1-7]. Based on the fact that nonlocal initial conditions are more effective than classical initial conditions in applied physics, the study of differential equations with nonlocal conditions has attracted more and more researchers' attention [8-13].

Ji and Li [14] studied the following impulsive differential evolution equations with nonlocal conditions:

$$
\left\{\begin{array}{l}
u^{\prime}(t)=A u(t)+f(t, u(t)), \quad t \in[0, b], t \neq t_{i}, \\
\left.\Delta u\right|_{t=t_{i}}=I_{i}\left(u\left(t_{i}\right)\right), \quad i=1,2, \cdots, m, \\
u(0)=g(u)
\end{array}\right.
$$

where $A$ is the generator of a strongly continuous semigroup $T_{\beta}(t)$; sufficient conditions for the existence of mild solutions have been established by the Hausdorff measure of noncompactness and fixed point theorems.
Zhu et al. [15] investigated the fractional semilinear integrodifferential equations of mixed type with nonlocal conditions:

$$
\left\{\begin{array}{l}
{ }^{c} D_{t}^{\beta} u(t)=A(t) u(t)+f(t, u(t), \mathscr{G} u(t), \delta u(t)), \quad t \in\left[0, T_{0}\right], \\
u(0)+g(u)=u_{0},
\end{array}\right.
$$

where $0<\beta \leq 1, A(t)$ is a closed linear operator with domain $D(A)$ defined on a Banach space $E$; the existence and uniqueness of mild solutions have been established by $k$-set contraction and $\beta$-resolvent family.

Gou and $\mathrm{Li}[16]$ studied the fractional impulsive integrodifferential equations in Banach space $E$; local and global existences of mild solutions have been proved by measure of noncompactness and Sadovskii's fixed point theorem:

$$
\left\{\begin{array}{l}
{ }^{c} D_{t}^{\beta} u(t)+A u(t)=f(t, u(t))+\int_{0}^{t} q(t-s) g(s, u(s)) d s, \quad t \geq 0, t \neq t_{k}, \\
\left.\Delta u\right|_{t=t_{k}}=I_{k}\left(u\left(t_{k}^{-}\right)\right), \quad k=1,2, \cdots, m, \\
u(0)=u_{0} \in E
\end{array}\right.
$$


where $0<\beta<1, A: D(A) \subset E \longrightarrow E$ is a closed linear operator and $-A$ generates a uniformly bounded $C_{0}$-semigroup $T(t)$.

Inspired by these contributions, we consider the following impulsive fractional semilinear integrodifferential equations with nonlocal initial conditions:

$$
\left\{\begin{array}{l}
{ }^{c} D_{t}^{\beta} x(t)-A(t) x(t)=f(t, x(t),(\mathscr{G} x)(t),(\mathscr{H} x)(t)), \quad t \in[0, T], t \neq t_{k}, \\
x(0)+w(x)=x_{0}, \\
\Delta x\left(t_{k}\right)=I_{k}\left(x\left(t_{k}\right)\right), \quad k=1,2, \cdots, m,
\end{array}\right.
$$

where ${ }^{c} D_{t}^{\beta}$ is the Caputo's fractional derivative of order $\beta, \beta$ $\in(0,1], A(t)$ is a closed linear operator with domain $D(A)$ defined on a Banach space $E$, and two integral operators $\mathscr{G}$ and $\mathscr{H}$ are defined by

$$
\begin{aligned}
& \mathscr{G} x(t)=\int_{0}^{t} g(t, s, x(s)) d s, \\
& \mathscr{H} x(t)=\int_{0}^{T} h(t, s, x(s)) d s
\end{aligned}
$$

$g: B \times E \longrightarrow E, h: B_{0} \times E \longrightarrow E$ are continuous and nonlinear functions, $B=\{(t, s) \mid 0 \leq s \leq t \leq T\}, B_{0}=\{(t, s) \mid$ $0 \leq t, s \leq T\}, f$ and $\omega$ are to be specified later, $I_{k}: E \longrightarrow E(k$ $=1,2, \cdots, m)$ are continuous impulsive functions, the prefixed numbers $t_{k}(k=1,2, \cdots, m)$ satisfy $0=t_{0}<t_{1}<t_{2}<\cdots<$ $t_{m}<t_{m+1}=T, x\left(t_{k}\right)=x\left(t_{k}^{-}\right)$, and $x\left(t_{k}^{-}\right)=\lim _{h \longrightarrow 0^{-}} x\left(t_{k}+h\right)$ represent the left limit of $x(t)$ at $t=t_{k}$.

In this paper, we demonstrate the existence of PC-mild solutions for problem (4) via the theory of semigroup and fixed point theorem under the condition of compact resolvent operator. Meanwhile, the uniqueness of PC-mild solutions is proved in the case of noncompact resolvent operator. The kernels $g$ and $h$ of the integral operators $\mathscr{G}$ and $\mathscr{H}$ are nonlinear functions; the function $\omega$ of the nonlocal conditions is noncompact. In addition, the closed linear operator $A(t)$ is dependent on $t$. The rest of this paper is organized as follows. In Section 2, some basic definitions and lemmas are collected that will be needed throughout the remaining sections. The existence and uniqueness of PC-mild solutions are shown in Section 3 via the theories of resolvent operators and various fixed point theorems. Finally, the summary of our results comes in Section 4.

\section{Preliminaries}

Let $(E,\|\cdot\|)$ be a Banach space, $J=[0, T]$ and $0<T<\infty$. The collection of all continuous functions from $J$ into $E$, denoted $C(J, E)$, is a Banach space equipped with the norm $\|x\|_{C}=$ $\max \{\|x(t)\|, t \in J\}$ for $x \in C(J, E)$. Let $P C(J, E)=\{x \mid x$ $: J \longrightarrow E: x \in C\left(\left(t_{k}, t_{k+1}\right], E\right)$, and there exist $x\left(t_{k}^{-}\right)$and $x\left(t_{k}^{+}\right)$ with $\left.x\left(t_{k}\right)=x\left(t_{k}^{-}\right), k=1, \cdots, m\right\}$ endowed with the PC-norm $\|x\|_{\mathrm{PC}}=\sup \{\|x(t)\|, t \in J\}, J_{0}=\left[0, t_{1}\right], J_{1}=\left(t_{1}, t_{2}\right], \cdots, J_{m}=$ $\left(t_{m}, T\right]$.
Lemma 1 (nonlinear alternative for single-valued maps). Let $E$ be a Banach space, $C \subset E$ be a closed convex set, $V$ be an open subset of $C$, and $0 \in V$. Suppose that $Q: \bar{V} \longrightarrow C$ is completely continuous, then either

(i) Q has a fixed point in $\bar{V}$ or

(ii) there is a $u \in \partial V$ and $\lambda \in(0,1)$ with $u=\lambda Q(u)$

Lemma 2 (see [17]). Let $0<\eta<1, \gamma>0$, denote

$$
S_{n}=\eta^{n}+C_{n}^{1} \eta^{n-1} \gamma+\frac{C_{n}^{2} \eta^{n-2}}{2 !} \gamma^{2}+\cdots+\frac{\gamma^{n}}{n !}, \quad n \in N,
$$

where $C_{n}^{k}=n ! /(k !(n-k) !)$. Then, for any fixed constant $0<$ $\xi<1$ and any real number $s>1$, we get

$$
S_{n} \leq O\left(\frac{\xi^{n}}{\sqrt{n}}\right)+o\left(\frac{1}{n^{s}}\right)=o\left(\frac{1}{n^{s}}\right) \text {, as } n \longrightarrow \infty \text {. }
$$

Definition 3 (see $[18,19])$. The Caputo fractional derivative of order $\beta$ of a function $f:(0, \infty) \longrightarrow R$ is defined as

$$
{ }^{c} D_{t}^{\beta} f(t)=\frac{1}{(\Gamma(n-\beta))} \int_{0}^{t}(t-s)^{n-\beta-1} f^{(n)}(s) d s
$$

where $n-1<\beta<n, n \in N, \Gamma(\cdot)$ denotes the Gamma function. The Laplace transform of the Caputo fractional derivative of order $\beta$ is given as

$$
\begin{gathered}
\mathscr{L}\left({ }^{c} D_{t}^{\beta} f(t)\right)(s)=s^{\beta}(\mathscr{L} f)(s)-\sum_{j=1}^{n-1} s^{\beta-j-1} x^{(j)}(0), \\
n-1<\beta \leq n,
\end{gathered}
$$

where $(\mathscr{L} f)(s)=\int_{0}^{\infty} e^{-s t} f(t) d t$ is the Laplace transform of the function $f(t)$.

Definition 4 (see $[20,21]$ ). Let $A(t)$ be a closed and linear operator with domain $D(A)$ defined on a Banach space $E$ and $\beta>0$. Let $\rho[A(t)]$ be the resolvent set of $A(t) ; A(t)$ is called the generator of a $\beta$-resolvent family if there exist $\omega$ $\geq 0$ and a strongly continuous function $U_{\beta}: \mathbb{R}_{+}^{2} \longrightarrow B(E)$ such that $\left\{\lambda^{\beta}: \operatorname{Re} \lambda>\omega\right\} \subset \rho(A)$ and

$$
\left(\lambda^{\beta} I-A(s)\right)^{-1} x=\int_{0}^{\infty} e^{-\lambda(t-s)} U_{\beta}(t, s) x d t, \quad \operatorname{Re}(\lambda)>\omega, x \in E .
$$

In this case, $U_{\beta}(t, s)$ is called the $\beta$-resolvent family generated by $A(t)$.

Lemma 5 (see $[21,22]) . U_{\beta}(t, s)$ satisfies the following properties: 
(i) $U_{\beta}(s, s)=I, U_{\beta}(t, s)=U_{\beta}(t, r) U_{\beta}(r, s)$, for $0 \leq s \leq r$ $\leq t \leq a$

(ii) $(t, s) \longrightarrow U_{\beta}(t, s)$ is strongly continuous for $0 \leq s \leq t$ $\leq a$

(iii) If $U_{\beta}(t, s)$ is compact for $t, s>0$, then the $U_{\beta}(t, s)$ is continuous in the uniform operator topology

Definition 6. A function $x \in P C(J, E)$ is said to be a PC-mild solution of problem (4) if $x(t)$ satisfies the integral equation:

$$
\begin{aligned}
x(t)= & U_{\beta}(t, 0)\left(x_{0}-\omega(x)\right) \\
& +\int_{0}^{t} U_{\beta}(t, s) f(s, x(s),(\mathscr{G} x)(s),(\mathscr{H} x)(s)) d s \\
& +\sum_{0<t_{k}<t} U_{\beta}\left(t, t_{k}\right) I_{k}\left(x\left(t_{k}\right)\right), \quad t \in J .
\end{aligned}
$$

\section{Existence and Uniqueness of Mild Solution}

Theorem 7. Assume that the conditions $\left(H_{1}\right)-\left(H_{3}\right)$ hold true and the resolvent operator $U_{\beta}(t, s)(t, s>0)$ is compact.

$\left(H_{1}\right)$ The function $f: J \times E \times E \times E \longrightarrow E$ is continuous, and there exist nonnegative Lebesgue integrable functions $a$, $l_{i} \in L\left(J, R_{+}\right)(i=1,2,3)$, for every $t \in J, x_{i} \in E$, such that

$$
\left\|f\left(t, x_{1}, x_{2}, x_{3}\right)\right\| \leq a(t)+l_{1}(t)\left\|x_{1}\right\|+l_{2}(t)\left\|x_{2}\right\|+l_{3}(t)\left\|x_{3}\right\| .
$$

$\left(\mathrm{H}_{2}\right)$ There exist nonnegative Lebesgue integrable functions $b, c, l_{i} \in L\left(J, \mathbb{R}_{+}\right)(i=4,5)$, for all $x \in E$, such that

$$
\begin{array}{ll}
\|g(t, s, x)\| \leq b(t)+l_{4}(t)\|x\|, & (t, s) \in B, \\
\|h(t, s, x)\| \leq c(t)+l_{5}(t)\|x\|, & (t, s) \in B_{0} .
\end{array}
$$

$\left(H_{3}\right)$ The functions $\omega: P C(J, E) \longrightarrow E$ and $I_{k}: E \longrightarrow E$ are continuous, and there exist constants $d_{\omega}, e_{\omega}, d_{k}, e_{k}>0$, such that

$$
\begin{aligned}
& \|\omega(x)\| \leq d_{\omega}\|x\|_{P C}+e_{\omega}, \quad x \in P C(J, E), \\
& \left\|I_{k}(x)\right\| \leq d_{k}\|x\|+e_{k}, \quad x \in E, k=1,2 \cdots m .
\end{aligned}
$$

Then, problem (4) has at least one PC-mild solution in $P C(J, E)$.

Proof. Let us consider the operator $Q: \mathrm{PC}(J, E) \longrightarrow \mathrm{PC}(J, E)$ as follows:

$$
\begin{aligned}
(Q x)(t)= & U_{\beta}(t, 0)\left(x_{0}-\omega(x)\right) \\
& +\int_{0}^{t} U_{\beta}(t, s) f(s, x(s),(\mathscr{G} x)(s),(\mathscr{H} x)(s)) d s \\
& +\sum_{0<t_{k}<t} U_{\beta}\left(t, t_{k}\right) I_{k}\left(x\left(t_{k}\right)\right) .
\end{aligned}
$$
$(J, E)$

It is easy to see that the operator $Q$ is well defined in PC

At first, we claim that $Q: \mathrm{PC}(J, E) \longrightarrow \mathrm{PC}(J, E)$ is a continuous operator. Let $\left\{x_{n}\right\}_{0}^{\infty} \subset \mathrm{PC}(J, E)$ be a sequence such that $x_{n} \longrightarrow x(n \longrightarrow \infty)$ in $\mathrm{PC}(J, E)$. Since for all $t \in J$,

$$
\begin{aligned}
& \left\|\left(Q x_{n}\right)(t)-(Q x)(t)\right\| \\
& \leq\left\|U_{\beta}(t, 0)\left(\omega\left(x_{n}\right)-\omega(x)\right)\right\| \\
& +\| \int_{0}^{t} \mathrm{U}_{\beta}(t, s)\left(f\left(s, x_{n}(s),\left(\mathscr{G} x_{n}\right)(s),\left(\mathscr{H} x_{n}\right)(s)\right)\right. \\
& \quad-f(s, x(s),(\mathscr{G} x)(s),(\mathscr{H} x)(s))) d s \| \\
& +\sum_{k=1}^{m}\left\|U_{\beta}\left(t, t_{k}\right)\left(I_{k}\left(x_{n}\left(t_{k}\right)\right)-I_{k}\left(x\left(t_{k}\right)\right)\right)\right\| \\
& \leq M\left\|\omega\left(x_{n}\right)-\omega(x)\right\|+M \int_{0}^{t} \| f\left(s, x_{n}(s),\left(\mathscr{G} x_{n}\right)(s),\right. \\
& \left.\quad \cdot\left(\mathscr{H} x_{n}\right)(s)\right)-f(s, x(s),(\mathscr{G} x)(s),(\mathscr{H} x)(s)) \| d s \\
& +\sum_{k=1}^{m} M\left\|I_{k}\left(x_{n}\left(t_{k}\right)\right)-I_{k}\left(x\left(t_{k}\right)\right)\right\|,
\end{aligned}
$$

where $M=\max _{0 \leq s \leq t \leq T}\left\|U_{\beta}(t, s)\right\|$. Using the fact that $f: J \times E$ $\times E \times E \longrightarrow E, \omega: \operatorname{PC}(J, E) \longrightarrow E$, and $I_{k}: E \longrightarrow E(k=1$, $2 \cdots m)$ are continuous, we obtain

$$
\left\|Q x_{n}-Q x\right\|_{\mathrm{PC}} \longrightarrow 0 \text {, as } n \longrightarrow \infty \text {. }
$$

Therefore, $Q: \mathrm{PC}(J, E) \longrightarrow \mathrm{PC}(J, E)$ is continuous.

Furthermore, for any $R>0$, we prove that $Q\left(T_{R}\right)$ is equicontinuous in $J_{k}(k=0,1,2 \cdots m)$. For all $x \in T_{R}=\{x \in$ $\left.\mathrm{PC}(J, E):\|x\|_{\mathrm{PC}} \leq R\right\}$ and $\tau_{1}, \tau_{2} \in J_{k}\left(\tau_{1} \leq \tau_{2}\right)$, by the condition $\left(H_{3}\right)$, we have

$$
\begin{aligned}
\|(Q x) & \left(\tau_{2}\right)-(Q x)\left(\tau_{1}\right) \| \\
\leq & \left\|U_{\beta}\left(\tau_{2}, 0\right)-U_{\beta}\left(\tau_{1}, 0\right)\right\|\left\|x_{0}-\omega(x)\right\| \\
& +\left\|\int_{\tau_{1}}^{\tau_{2}} U_{\beta}\left(\tau_{2}, s\right) f(s, x(s),(\mathscr{G} x)(s),(\mathscr{H} x)(s)) d s\right\| \\
& +\left\|\int_{0}^{\tau_{1}}\left(U_{\beta}\left(\tau_{2}, s\right)-U_{\beta}\left(\tau_{1}, s\right)\right) f(s, x(s),(\mathscr{G} x)(s),(\mathscr{H} x)(s)) d s\right\| \\
& +\left\|\sum_{0<t_{k}<\tau_{2}} U_{\beta}\left(\tau_{2}, t_{k}\right) I_{k}\left(x\left(t_{k}\right)\right)-\sum_{0<t_{k}<\tau_{1}} U_{\beta}\left(\tau_{1}, t_{k}\right) I_{k}\left(x\left(t_{k}\right)\right)\right\| \\
\leq & \left.\| U_{\beta}\left(\tau_{2}, 0\right)-U_{\beta}\left(\tau_{1}, 0\right)\right) \|\left(\left\|x_{0}\right\|+d_{\omega} R+e_{\omega}\right) \\
& +\int_{\tau_{1}}^{\tau_{2}} M\|f(s, x(s),(\mathscr{G} x)(s),(\mathscr{H} x)(s))\| d s \\
& +\sup _{s \in J_{k}}\left\|U_{\beta}\left(\tau_{2}, s\right)-U_{\beta}\left(\tau_{1}, s\right)\right\| \\
& \cdot \int_{0}^{\tau_{1}}\|f(s, x(s),(\mathscr{G} x)(s),(\mathscr{H} x)(s))\| d s \\
& +\sum_{k=1}^{m}\left\|U_{\beta}\left(\tau_{2}, t_{k}\right)-U_{\beta}\left(\tau_{1}, t_{k}\right)\right\|\left\|I_{k}\left(x\left(t_{k}\right)\right)\right\|=: I_{1}+I_{2}+I_{3}+I_{4} .
\end{aligned}
$$



$\left(\mathrm{H}_{2}\right)$,

For all $x \in T_{R}, s \in J$, we get $\|x(s)\| \leq R$, by the condition

$$
\begin{aligned}
\|(\mathscr{G} x)(s)\| & \leq \int_{0}^{s}\|g(s, v, x(v))\| d v \leq \int_{0}^{s}\left(b(v)+l_{4}(v)\|x(v)\|\right) d v \\
& \leq \int_{0}^{T} b(v) d v+R \int_{0}^{T} l_{4}(v) d v,
\end{aligned}
$$

meanwhile,

$$
\begin{aligned}
\|(\mathscr{H} x)(s)\| & \leq \int_{0}^{T}\|h(s, v, x(v))\| d v \leq \int_{0}^{T}\left(c(v)+l_{5}(v)\|x(v)\|\right) d v \\
& \leq \int_{0}^{T} c(v) d v+R \int_{0}^{T} l_{5}(v) d v .
\end{aligned}
$$

According to the condition $\left(H_{1}\right)$ and the above inequalities, for all $s \in J$, we get

$$
\begin{aligned}
& \|f(s, x(s),(\mathscr{G} x)(s),(\mathscr{H} x)(s))\| \\
& \leq a(s)+l_{1}(s)\|x(s)\|+l_{2}(s)\|(\mathscr{G} x)(s)\|+l_{3}(s)\|(\mathscr{H} x)(s)\| \\
& \leq a(s)+l_{1}(s) R+l_{2}(s)\left(\int_{0}^{T} b(v) d v+R \int_{0}^{T} l_{4}(v) d v\right) \\
& \quad+l_{3}(s)\left(\int_{0}^{T} c(v) d v+R \int_{0}^{T} l_{5}(v) d v\right) \leq a_{1}(s)+b_{1}(s) R,
\end{aligned}
$$

where

$$
\begin{aligned}
& a_{1}(s)=a(s)+l_{2}(s) \int_{0}^{T} b(v) d v+l_{3}(s) \int_{0}^{T} c(v) d v, \\
& b_{1}(s)=l_{1}(s)+l_{2}(s) \int_{0}^{T} l_{4}(v) d v+l_{3}(s) \int_{0}^{T} l_{5}(v) d v .
\end{aligned}
$$

Obviously, $a_{1}(s)$ and $b_{1}(s)$ are nonnegative Lebesgue integrable functions, then

$$
\begin{aligned}
I_{2}= & \int_{\tau_{1}}^{\tau_{2}} M\|f(s, x(s),(\mathscr{G} x)(s),(\mathscr{H} x)(s))\| d s \\
\leq & M \int_{\tau_{1}}^{\tau_{2}}\left(a_{1}(s)+b_{1}(s) R\right) d s, \\
I_{3}= & \sup _{s \in J_{k}}\left\|U_{\beta}\left(\tau_{2}, s\right)-U_{\beta}\left(\tau_{1}, s\right)\right\| \\
& \cdot \int_{0}^{\tau_{1}}\|f(s, x(s),(\mathscr{G} x)(s),(\mathscr{H} x)(s))\| d s \\
\leq & \sup _{s \in J_{k}}\left\|U_{\beta}\left(\tau_{2}, s\right)-U_{\beta}\left(\tau_{1}, s\right)\right\| \int_{0}^{\tau_{1}}\left(a_{1}(s)+b_{1}(s) R\right) d s,
\end{aligned}
$$

$$
\begin{aligned}
I_{4} & =\sum_{k=1}^{\mathrm{m}}\left\|U_{\beta}\left(\tau_{2}, t_{k}\right)-U_{\beta}\left(\tau_{1}, t_{k}\right)\right\|\left\|I_{k}\left(x\left(t_{k}\right)\right)\right\| \\
& \leq \sum_{k=1}^{m}\left\|U_{\beta}\left(\tau_{2}, t_{k}\right)-U_{\beta}\left(\tau_{1}, t_{k}\right)\right\|\left(d_{k} R+e_{k}\right) .
\end{aligned}
$$

In view of Lemma 5 , the compactness of the resolvent operator $U_{\beta}(t, s)(t, s>0)$ implies the continuity in the uniform operator topology. As a result, from the above inequalities, we deduce that $\left\|(Q x)\left(\tau_{2}\right)-(Q x)\left(\tau_{1}\right)\right\| \longrightarrow 0$ independently of $x \in T_{R}$ as $\tau_{2}-\tau_{1} \longrightarrow 0$. That is, $Q\left(T_{R}\right)$ is equicontinuous in $J_{k}(k=0,1,2 \cdots m)$.

In the end, we demonstrate that $Q\left(T_{R}\right) \subset \mathrm{PC}(J, E)$ is precompact.

For any $t(0<t \leq T), 0<\varepsilon<t$, and $x \in T_{R}$, the operator $Q_{\varepsilon} x$ is defined by

$$
\begin{aligned}
\left(Q_{\varepsilon} x\right)(t)= & U_{\beta}(t, 0)\left(x_{0}-\omega(x)\right) \\
& +\int_{0}^{t-\varepsilon} U_{\beta}(t, s) f(s, x(s),(\mathscr{G} x)(s),(\mathscr{H} x)(s)) d s \\
& +\sum_{0<t_{k}<t} U_{\beta}\left(t, t_{k}\right) I_{k}\left(x\left(t_{k}\right)\right), \quad t \in J .
\end{aligned}
$$

Since $U_{\beta}(t, s)$ is compact resolvent operator, the set $Y_{\varepsilon}(t)=\left\{\left(Q_{\varepsilon} x\right)(t): x \in T_{R}\right\}$ is relatively compact in $E$ for every $\varepsilon(0<\varepsilon<t)$.

Moreover, for any $x \in T_{R}, t \in J$, one can show that

$$
\begin{aligned}
& \left\|(Q x)(t)-\left(Q_{\varepsilon} x\right)(t)\right\| \\
& \quad=\left\|\int_{t-\varepsilon}^{t} U_{\beta}(t, s) f(s, x(s),(\mathscr{G} x)(s),(\mathscr{H} x)(s)) d s\right\| \\
& \leq M \int_{t-\varepsilon}^{t} \| f(s, x(s,(\mathscr{G} x)(s),(\mathscr{H} x)(s)) \| d s \\
& \leq M \int_{t-\varepsilon}^{t}\left(a_{1}(s)+b_{1}(s) R\right) d s .
\end{aligned}
$$

Thus, $Y(t)=\left\{(Q x)(t): x \in T_{R}\right\} \quad$ is totally bounded. Hence, $Y(t)$ is relatively compact in $E$, and so, with the help of the Arzelà-Ascoli theorem, $Q: \mathrm{PC}(J, E) \longrightarrow \mathrm{PC}(J, E)$ is completely continuous.

For $0<\lambda<1$, let $x=\lambda(Q x)$, we get

$$
\begin{aligned}
x(t)= & \lambda U_{\beta}(t, 0)\left(x_{0}-\omega(x)\right) \\
& +\lambda \int_{0}^{t} U_{\beta}(t, s) f(s, x(s),(\mathscr{G} x)(s),(\mathscr{H} x)(s)) d s \\
& +\lambda \sum_{0<t_{k}<t} U_{\beta}\left(t, t_{k}\right) I_{k}\left(x\left(t_{k}\right)\right) .
\end{aligned}
$$


Then, using the conditions $\left(H_{1}\right)-\left(H_{3}\right)$, it follows that

$$
\begin{aligned}
&\|x(t)\| \leq M\left\|x_{0}-\omega(x)\right\|+M \int_{0}^{t} \| f(s, x(s), \\
&\cdot(\mathscr{G} x)(s),(\mathscr{H} x)(s))\left\|d s+\sum_{k=1}^{m} M\right\| I_{k}\left(x\left(t_{k}\right)\right) \| \\
& \leq M\left(\left\|x_{0}\right\|+d_{\omega} R+e_{\omega}\right)+M \int_{0}^{t}\left(a_{1}(s)\right. \\
&+\left.b_{1}(s) R\right) d s+\sum_{k=1}^{m} M\left(d_{k} R+e_{k}\right) \\
& \leq M\left(\left\|x_{0}\right\|+e_{\omega}\right)+M \int_{0}^{T} a_{1}(s) d s+\sum_{k=1}^{m} M e_{k} \\
&+\left(M d_{\omega}+M \int_{0}^{T} b_{1}(s) d s+\sum_{k=1}^{m} M d_{k}\right) R=: \rho .
\end{aligned}
$$

That is, $\|x(t)\| \leq \rho$ for $t \in J$, then there exists a constant $\rho_{1}>\rho$ such that $\|x\|_{\mathrm{PC}} \neq \rho_{1}$. Let $V=\left\{x \in \mathrm{PC}(J, E):\|x\|_{\mathrm{PC}}<\right.$ $\left.\rho_{1}\right\}$, obviously, there is no $x \in \partial V$ such that $x=\lambda(Q x)$ for 0 $<\lambda<1$. Therefore, thanks to Lemma 1, one gets that $Q$ has at least one fixed point $x$ in $V$, which is a PC-mild solution of problem (4). This completes the proof.

Remark 8. Theorem 7 is proved under the condition that $U_{\beta}(t, s)$ is compact for $t, s>0$ and the functions $f, g, h$ meet corresponding conditions; in the case that the resolvent operator $U_{\beta}(t, s)$ is noncompact, we would obtain Theorem 9 and Theorem 10.

Theorem 9. Suppose that the conditions $\left(H_{4}\right)-\left(H_{6}\right)$ are satisfied, $M=\max _{0 \leq s<t \leq T}\left\|U_{\beta}(t, s)\right\|$, and $M\left(L_{\omega}+\int_{0}^{T} L_{1}(s) d s+\int_{0}^{T} L_{2}(s)\right.$ $\left.d s \int_{0}^{T} L_{4}(v) d v+\int_{0}^{T} L_{3}(s) d s \int_{0}^{T} L_{5}(v) d v+\sum_{k=1}^{m} L_{I_{k}}\right)<1$.

$\left(\mathrm{H}_{4}\right)$ The function $f: J \times E \times E \times E \longrightarrow E$ is continuous, and there exist nonnegative Lebesgue integrable functions $L_{i}$ $\in L\left(J, \mathbb{R}_{+}\right)(i=1,2,3)$, for any $u_{i}, v_{i} \in E, t \in J$, such that

$$
\begin{aligned}
& \left\|f\left(t, u_{1}, u_{2}, u_{3}\right)-f\left(t, v_{1}, v_{2}, v_{3}\right)\right\| \\
& \quad \leq L_{1}(t)\left\|u_{1}-v_{1}\right\|+L_{2}(t)\left\|u_{2}-v_{2}\right\|+L_{3}(t)\left\|u_{3}-v_{3}\right\| .
\end{aligned}
$$

$\left(H_{5}\right)$ There exist nonnegative Lebesgue integrable functions $L_{4}, L_{5} \in L\left(J, \mathbb{R}_{+}\right)$, for each $u, v \in E$, such that

$$
\begin{aligned}
\|g(t, s, u)-g(t, s, v)\| \leq L_{4}(t)\|u-v\|, & (t, s) \in B, \\
\|h(t, s, u)-h(t, s, v)\| \leq L_{5}(t)\|u-v\|, & (t, s) \in B_{0} .
\end{aligned}
$$

$\left(H_{6}\right)$ The functions $I_{k}: E \longrightarrow E$ and $\omega: P C(J, E) \longrightarrow E$ are continuous, and there exist nonnegative constants $L_{\omega}, L_{I_{k}}$ $>0$, such that

$$
\begin{aligned}
& \|\omega(u)-\omega(v)\| \leq L_{\omega}\|u-v\|_{P C}, \quad u, v \in P C(J, E), \\
& \left\|I_{k}(u)-I_{k}(v)\right\| \leq L_{I_{k}}\|u-v\|, \quad u, v \in E, k=1,2 \cdots m .
\end{aligned}
$$

Then, problem (4) has a unique PC-mild solution $x^{\star}$ in $P C(J, E)$.

Proof. It follows from the conditions $\left(H_{4}\right)-\left(H_{6}\right)$, for any $u$, $v \in \mathrm{PC}(J, E), t \in J$, one can derive

$$
\begin{aligned}
& \|(Q u)(t)-(Q v)(t)\| \\
& \leq\left\|U_{\beta}(t, 0)(\omega(u)-\omega(v))\right\| \\
& +\sum_{0<t_{k}<t}\left\|U_{\beta}\left(t, t_{k}\right)\left(I_{k}\left(u\left(t_{k}\right)\right)-I_{k}\left(v\left(t_{k}\right)\right)\right)\right\| \\
& +\| \int_{0}^{t} U_{\beta}(t, s)(f(s, u(s),(\mathscr{G} u)(s),(\mathscr{H} u)(s)) \\
& -f(s, v(s),(\mathscr{G} v)(s),(\mathscr{H} v)(s))) d s \| \\
& \leq M\|\omega(u)-\omega(v)\|+\sum_{k=1}^{m} M\left\|I_{k}\left(u\left(t_{k}\right)\right)-I_{k}\left(v\left(t_{k}\right)\right)\right\| \\
& +M \int_{0}^{t} \| f(s, u(s),(\mathscr{G} u)(s),(\mathscr{H} u)(s)) \\
& -f(s, v(s),(\mathscr{G} v)(s),(\mathscr{H} v)(s)) \| d s \\
& \leq M L_{\omega}\|u-v\|_{\mathrm{PC}}+\sum_{k=1}^{m} M L_{I_{k}}\left\|u\left(t_{k}\right)-v\left(t_{k}\right)\right\| \\
& +M \int_{0}^{t}\left(L_{1}(s)\|u(s)-v(s)\|+L_{2}(s)\|(\mathscr{G} u)(s)-(\mathscr{G} v)(s)\|\right. \\
& \left.+L_{3}(s)\|(\mathscr{H} u)(s)-(\mathscr{H} v)(s)\|\right) d s \\
& \leq M L_{\omega}\|u-v\|_{\mathrm{PC}}+\sum_{k=1}^{m} M L_{I_{k}}\|u-v\|_{\mathrm{PC}} \\
& +M \int_{0}^{t} L_{1}(s) d s\|u-v\|_{\mathrm{PC}}+\left(M \int_{0}^{t} L_{2}(s) \int_{0}^{s} L_{4}(v) d v d s\right. \\
& \left.+M \int_{0}^{t} L_{3}(s) \int_{0}^{T} L_{5}(v) d v d s\right)\|u-v\|_{\mathrm{PC}} \\
& \leq M\left(L_{\omega}+\sum_{k=1}^{m} L_{I_{k}}+\int_{0}^{T} L_{1}(s) d s+\int_{0}^{T} L_{2}(s) d s \int_{0}^{T} L_{4}(v) d v\right. \\
& \left.+\int_{0}^{T} L_{3}(s) d s \int_{0}^{T} L_{5}(v) d v\right)\|u-v\|_{\mathrm{PC}} .
\end{aligned}
$$

Based on the assumption, we have $\|Q u-Q v\|_{\mathrm{PC}}<$ $\|u-v\|_{\mathrm{PC}}$, which means that the operator $Q$ is a contraction mapping. Hence, the operator $Q$ has a unique fixed point $x^{\star} \in \mathrm{PC}(J, E)$, which implies that problem (4) has a unique PC-mild solution. This completes the proof.

Theorem 10. Assume that the conditions $\left(H_{7}\right)$ and $\left(H_{8}\right)$ hold, $\omega \equiv 0, M=\max _{0 \leq s<t \leq T}\left\|U_{\beta}(t, s)\right\|, a=\sum_{k=1}^{m} M L_{I_{k}}<1$. 
$\left(H_{7}\right)$ The function $f: J \times E \times E \times E \longrightarrow E$ is continuous, and there exist nonnegative Lebesgue integrable functions $L$ ${ }_{1}^{1}, L^{\prime}{ }_{2} \in L\left(J, \mathbb{R}_{+}\right)$, for all $u_{i}, v_{i} \in E, t \in J$, such that

$$
\begin{aligned}
& \left\|f\left(t, u_{1}, u_{2}, u_{3}\right)-f\left(t, v_{1}, v_{2}, v_{3}\right)\right\| \\
& \quad \leq L^{\prime}{ }_{1}(t)\left\|u_{1}-v_{1}\right\|+L^{\prime}{ }_{2}(t)\left\|u_{2}-v_{2}\right\| .
\end{aligned}
$$

$\left(H_{8}\right)$ There exist constants $L_{g}, L_{I_{k}}>0$, for all $u, v \in E$, satisfying

$$
\begin{array}{rlrl}
\|g(t, s, u)-g(t, s, v)\| & \leq L_{g}\|u-v\|, & & (t, s) \in B, \\
\left\|I_{k}(u)-I_{k}(v)\right\| & \leq L_{I_{k}}\|u-v\|, & k=1,2 \cdots m .
\end{array}
$$

Then, problem (4) has a unique PC-mild solution $x^{\star}$ in $P C(J, E)$. For all $t \in J, x_{0} \in P C(J, E)$, iterative sequence $x_{n}(t)$ are defined by

$$
\begin{aligned}
x_{n}(t)= & U_{\beta}(t, 0) x_{0}+\int_{0}^{t} U_{\beta}(t, s) f\left(s, x_{n-1}(s),\right. \\
& \left.\cdot\left(\mathscr{G} x_{n-1}\right)(s),\left(\mathscr{H} x_{n-1}\right)(s)\right) d s \\
& +\sum_{0<t_{k}<t} U_{\beta}\left(t, t_{k}\right) I_{k}\left(x_{n-1}\left(t_{k}\right)\right), \quad n=1,2, \cdots,
\end{aligned}
$$

uniformly converge to the unique PC-mild solution $x^{\star}(t)$ in $t \in J$, and for any $s>0$,

$$
\left\|x_{n}-x^{\star}\right\|_{P C}=o\left(\frac{1}{n^{s}}\right) \text {, as } n \longrightarrow \infty .
$$

Proof. Combining the conditions $\left(H_{7}\right)$ and $\left(H_{8}\right)$, for all $t \in J$, $u, v \in P C(J, E)$, we get

$$
\begin{aligned}
& \|(\mathrm{Qu})(t)-(\mathrm{Q} v)(t)\| \\
& \leq M \int_{0}^{t} \| f(s, u(s),(\mathscr{G} u)(s),(\mathscr{H} u)(s)) \\
& \quad-f(s, v(s),(\mathscr{G} v)(s),(\mathscr{H} v)(s)) \| d s \\
& \quad+\sum_{0<t_{k}<t} M\left\|I_{k}\left(u\left(t_{k}\right)\right)-I_{k}\left(v\left(t_{k}\right)\right)\right\| \\
& \leq M \int_{0}^{t}\left(L_{1}^{\prime}(s)+L_{2}^{\prime}(s) L_{g} s\right)\|u(s)-v(s)\| d s \\
& \quad+\sum_{k=1}^{m} M L_{I_{k}}\left\|u\left(t_{k}\right)-v\left(t_{k}\right)\right\| \leq \int_{0}^{t} L(s)\|u(s)-v(s)\| d s \\
& \quad+\sum_{k=1}^{m} M L_{I_{k}}\|u-v\|_{\mathrm{PC}} \leq\left(\int_{0}^{t} L(s) d s+\sum_{k=1}^{m} M L_{I_{k}}\right)\|u-v\|_{\mathrm{PC}} \\
& \leq\left(\int_{0}^{t} L(s) d s+a\right)\|u-v\|_{\mathrm{PC}}
\end{aligned}
$$

where $L(s)=M\left(L_{1}^{\prime}(s)+L_{2}^{\prime}(s) L_{g} T\right)$. It is easy to see that $L \epsilon$ $L\left(J, \mathbb{R}_{+}\right)$. Notice that $a=\sum_{k=1}^{m} M L_{I_{k}}<1$, then there is $\varepsilon>0$ such that $0<b=\varepsilon+a<1$. For the above $\varepsilon>0$, there exists a continuous function $\phi(s)$ such that

$$
\int_{0}^{T}|L(s)-\phi(s)| d s<\varepsilon
$$

Consequently,

$$
\begin{aligned}
\|(\mathrm{Q} u)(t)-(\mathrm{Q} v)(t)\| \\
\quad \leq\left(\int_{0}^{t}|L(s)-\phi(s)| d s+\int_{0}^{t}|\phi(s)| d s\right)\|u-v\|_{\mathrm{PC}}+a\|u-v\|_{\mathrm{PC}} \\
\quad \leq(\varepsilon+\Phi t)\|u-v\|_{\mathrm{PC}}+a\|u-v\|_{\mathrm{PC}} \leq(b+\Phi t)\|u-v\|_{\mathrm{PC}} \\
\quad=\left(C_{1}^{0} b+C_{1}^{1} \frac{(\Phi t)}{1 !}\right)\|u-v\|_{\mathrm{PC}},
\end{aligned}
$$

where $\Phi=\max \{|\phi(s)|: s \in J\}$. We next prove the following inequalities, for every positive integer $n$ and $t \in J$,

$$
\begin{aligned}
& \left\|\left(Q^{n} u\right)(t)-\left(Q^{n} v\right)(t)\right\| \\
& \leq\left(C_{n}^{0} b^{n}+C_{n}^{1} \frac{b^{n-1}(\Phi t)}{1 !}+C_{n}^{2} \frac{b^{n-2}(\Phi t)^{2}}{2 !}\right. \\
& \left.\quad+\cdots+C_{n}^{n} \frac{b^{0}(\Phi t)^{n}}{n !}\right)\|u-v\|_{\mathrm{PC}},
\end{aligned}
$$

where $C_{n}^{m}=n ! /(m !(n-m) !)$.

Assume that, for any positive integer $k$, we have

$$
\begin{aligned}
\left\|\left(Q^{k} u\right)(t)-\left(Q^{k} v\right)(t)\right\| \\
\leq\left(C_{k}^{0} b^{k}+C_{k}^{1} \frac{b^{k-1}(\Phi t)}{1 !}+C_{k}^{2} \frac{b^{k-2}(\Phi t)^{2}}{2 !}\right. \\
\left.\quad+\cdots+C_{k}^{k} \frac{b^{0}(\Phi t)^{k}}{k !}\right)\|u-v\|_{\mathrm{PC} .}
\end{aligned}
$$

By the formula $C_{k+1}^{m}=C_{k}^{m}+C_{k}^{m-1}$, for all $t \in J$,

$$
\begin{aligned}
& \left\|\left(Q^{k+1} u\right)(t)-\left(Q^{k+1} v\right)(t)\right\| \\
& =\left\|Q\left(Q^{k} u\right)(t)-Q\left(Q^{k} v\right)(t)\right\| \\
& \leq M \int_{0}^{t} \| f\left(s,\left(Q^{k} u\right)(s), \mathscr{G}\left(Q^{k} u\right)(s), \mathscr{H}\left(Q^{k} u\right)(s)\right) \\
& \quad-f\left(s,\left(Q^{k} v\right)(s), \mathscr{G}\left(Q^{k} v\right)(s), \mathscr{H}\left(Q^{k} v\right)(s)\right) \| d s \\
& \quad+\sum_{0<t_{k}<t} M\left\|I_{k}\left(Q^{k} u\right)\left(t_{k}\right)-I_{k}\left(Q^{k} v\right)\left(t_{k}\right)\right\| \\
& \leq M \int_{0}^{t}\left(L_{1}^{\prime}(s)+L_{2}^{\prime}(s) L_{g} s\right)\left\|\left(Q^{k} u\right)(s)-\left(Q^{k} v\right)(s)\right\| d s \\
& \quad+\sum_{k=1}^{m} M L_{I_{k}}\left\|\left(Q^{k} u\right)\left(t_{k}\right)-\left(Q^{k} v\right)\left(t_{k}\right)\right\|
\end{aligned}
$$




$$
\begin{aligned}
& \leq \int_{0}^{t} L(s)\left\|\left(Q^{k} u\right)(s)-\left(Q^{k} v\right)(s)\right\| d s \\
& +\sum_{k=1}^{m} M L_{I_{k}}\left\|\left(Q^{k} u\right)\left(t_{k}\right)-\left(Q^{k} v\right)\left(t_{k}\right)\right\| \\
& \leq \int_{0}^{t}|L(s)-\phi(s)|\left(C_{k}^{0} b^{k}+C_{k}^{1} \frac{b^{k-1}(\Phi s)}{1 !}\right. \\
& \left.+C_{k}^{2} \frac{b^{k-2}(\Phi s)^{2}}{2 !}+\cdots+C_{k}^{k} \frac{b^{0}(\Phi s)^{k}}{k !}\right) d s\|u-\mathrm{v}\|_{\mathrm{PC}} \\
& +\int_{0}^{t}|\phi(s)|\left(C_{k}^{0} b^{k}+C_{k}^{1} \frac{b^{k-1}(\Phi s)}{1 !}\right. \\
& \left.+C_{k}^{2} \frac{b^{k-2}(\Phi s)^{2}}{2 !}+\cdots+C_{k}^{k} \frac{b^{0}(\Phi s)^{k}}{k !}\right) d s\|u-v\|_{\mathrm{PC}} \\
& +\sum_{k=1}^{m} M L_{I_{k}}\left(C_{k}^{0} b^{k}+C_{k}^{1} \frac{b^{k-1}\left(\Phi t_{k}\right)}{1 !}\right. \\
& \left.+C_{k}^{2} \frac{b^{k-2}\left(\Phi t_{k}\right)^{2}}{2 !}+\cdots+C_{k}^{k} \frac{b^{0}\left(\Phi t_{k}\right)^{k}}{k !}\right)\|u-v\|_{\mathrm{PC}} \\
& \leq \varepsilon\left(C_{k}^{0} b^{k}+C_{k}^{1} \frac{b^{k-1}(\Phi t)}{1 !}+C_{k}^{2} \frac{b^{k-2}(\Phi t)^{2}}{2 !}\right. \\
& \left.+\cdots+C_{k}^{k} \frac{b^{0}(\Phi t)^{k}}{k !}\right)\|u-v\|_{\mathrm{PC}}+\Phi \int_{0}^{t}\left(C_{k}^{0} b^{k}\right. \\
& +C_{k}^{1} \frac{b^{k-1}(\Phi s)}{1 !}+C_{k}^{2} \frac{b^{k-2}(\Phi s)^{2}}{2 !}+\cdots \\
& +C_{k}^{k} \frac{b^{0}(\Phi s)^{k}}{k !} d s\|u-v\|_{\mathrm{PC}}+a\left(C_{k}^{0} b^{k}+C_{k}^{1} \frac{b^{k-1}(\Phi t)}{1 !}\right. \\
& \left.+C_{k}^{2} \frac{b^{k-2}(\Phi t)^{2}}{2 !}+\cdots+C_{k}^{k} \frac{b^{0}(\Phi t)^{k}}{k !}\right)\|u-v\|_{\mathrm{PC}} \\
& \leq b\left(C_{k}^{0} b^{k}+C_{k}^{1} \frac{b^{k-1}(\Phi t)}{1 !}+C_{k}^{2} \frac{b^{k-2}(\Phi t)^{2}}{2 !}+\cdots\right. \\
& \left.+C_{k}^{k} \frac{b^{0}(\Phi t)^{k}}{k !}\right)\|u-v\|_{\mathrm{PC}}+\Phi \int_{0}^{t}\left(C_{k}^{0} b^{k}+C_{k}^{1} \frac{b^{k-1}(\Phi s)}{1 !}\right. \\
& \left.+C_{k}^{2} \frac{b^{k-2}(\Phi s)^{2}}{2 !}+\cdots+C_{k}^{k} \frac{b^{0}(\Phi s)^{k}}{k !}\right) d s\|u-v\|_{\mathrm{PC}} \\
& \leq\left(C_{k+1}^{0} b^{k+1}+C_{k+1}^{1} \frac{b^{k}(\Phi t)}{1 !}+C_{k+1}^{2} \frac{b^{k-1}(\Phi t)^{2}}{2 !}\right. \\
& \left.+\cdots+C_{k+1}^{k+1} \frac{b^{0}(\Phi t)^{k+1}}{(k+1) !}\right)\|u-v\|_{\mathrm{PC}} .
\end{aligned}
$$

By mathematical induction, for every positive integer $n$, we obtain

$$
\begin{aligned}
\left\|Q^{n} u-Q^{n} v\right\|_{\mathrm{PC}} \leq & \left(C_{n}^{0} b^{n}+C_{n}^{1} \frac{b^{n-1}(\Phi t)}{1 !}+C_{n}^{2} \frac{b^{n-2}(\Phi t)^{2}}{2 !}\right. \\
& \left.+\cdots+C_{n}^{n} \frac{b^{0}(\Phi t)^{n}}{n !}\right)\|u-v\|_{\mathrm{PC}} .
\end{aligned}
$$

Using Lemma 2, it follows that

$$
\left\|Q^{n} u-Q^{n} v\right\|_{\mathrm{PC}} \leq o\left(\frac{1}{n^{s}}\right)\|u-v\|_{\mathrm{PC}} \text {, as } n \longrightarrow \infty \text {. }
$$

Thus, for any fixed constant $s>1$, we can find a positive integer $n_{0}$ such that, for any $u, v \in \operatorname{PC}(J, E)$ and $n>n_{0}$, we have

$$
\left\|Q^{n} u-Q^{n} v\right\|_{\mathrm{PC}} \leq \frac{1}{n^{s}}\|u-v\|_{\mathrm{PC}} .
$$

Applying the general Banach contraction mapping principle, we deduce that the operator $Q$ has a unique fixed point $x^{\star}$ in $\operatorname{PC}(J, E)$, which means that problem (4) has a unique PC-mild solution $x^{\star}$ in $\operatorname{PC}(J, E)$. This completes the proof.

\section{Conclusion}

In this paper, we demonstrate the existence theory of PCmild solutions for the impulsive fractional semilinear integrodifferential equations with nonlocal initial conditions (4) via the theory of semigroup and fixed point theorem under the condition of compact resolvent operator. Meanwhile, the uniqueness of PC-mild solutions is proved under the condition of noncompact resolvent operator. The kernels $g$ and $h$ of the integral operators $\mathscr{G}$ and $\mathscr{H}$ are nonlinear functions, and the function $\omega$ of the nonlocal initial conditions is noncompact. In addition, the closed linear operator $A(t)$ is dependent on $t$. As a consequence, our main theorems improve and generalize many existing results on this topic.

\section{Data Availability}

No data were used to support this study.

\section{Conflicts of Interest}

The authors declare that they have no conflicts of interest.

\section{Authors' Contributions}

All authors contributed equally and significantly in writing this article. All authors read and approved the final manuscript.

\section{Acknowledgments}

This research was supported by the Project of Shandong Province Higher Educational Science and Technology Program (No. J16LI14). 


\section{References}

[1] H. Li and Y. Kao, "Mittag-Leffler stability for a new coupled system of fractional-order differential equations with impulses," Applied Mathematics and Computation, vol. 361, pp. 22-31, 2019.

[2] A. Chadha and D. N. Pandey, "Existence results for an impulsive neutral stochastic fractional integro- differential equation with infinite delay," Nonlinear Analysis, vol. 128, pp. 149-175, 2015.

[3] G. Arthi, J. H. Park, and H. Y. Jung, "Existence and exponential stability for neutral stochastic integrodifferential equations with impulses driven by a fractional Brownian motion," Communications in Nonlinear Science and Numerical Simulation, vol. 32, pp. 145-157, 2016.

[4] A. Chauhan and J. Dabas, "Local and global existence of mild solution to an impulsive fractional functional integrodifferential equation with nonlocal condition," Communications in Nonlinear Science and Numerical Simulation, vol. 19, no. 4, pp. 821-829, 2014.

[5] Z. Yan and F. Lu, "Approximate controllability of a multivalued fractional impulsive stochastic partial integrodifferential equation with infinite delay," Applied Mathematics and Computation, vol. 292, pp. 425-447, 2017.

[6] Y. Liu, "Piecewise continuous solutions of initial value problems of singular fractional differential equations with impulse effects," Acta Mathematica Scientia, vol. 36, no. 5, pp. 14921508, 2016.

[7] F. D. Ge, H. C. Zhou, and C. H. Kou, "Approximate controllability of semilinear evolution equations of fractional order with nonlocal and impulsive conditions via an approximating technique," Applied Mathematics and Computation, vol. 275, pp. 107-120, 2016.

[8] P. Chen, X. Zhang, and Y. Li, "Approximate controllability of non-autonomous evolution system with nonlocal conditions," Journal of Dynamical and Control Systems, vol. 26, no. 1, pp. 1-16, 2020.

[9] P. Chen, X. Zhang, and Y. Li, "Existence and approximate controllability of fractional evolution equations with nonlocal conditions via resolvent operators," Fractional Calculus and Applied Analysis, vol. 23, no. 1, pp. 268-291, 2020.

[10] R. Agarwal, D. Baleanu, J. J. Nieto, D. F. M. Torres, and Y. Zhou, "A survey on fuzzy fractional differential and optimal control nonlocal evolution equations," Journal of Computational and Applied Mathematics, vol. 339, pp. 3-29, 2018.

[11] X. Shu and Q. Wang, "The existence and uniqueness of mild solutions for fractional differential equations with nonlocal conditions of order $1<\beta<2$," Computers and Mathematics with Applications, vol. 64, no. 6, pp. 2100-2110, 2012.

[12] P. Chen, X. Zhang, and Y. Li, "Fractional non-autonomous evolution equation with nonlocal conditions," Journal of Pseudo-Differential Operators and Applications, vol. 10, no. 4, pp. 955-973, 2019.

[13] P. Chen and X. Zhang, "Non-autonomous stochastic evolution equations of parabolic type with nonlocal initial conditions," Discrete \& Continuous Dynamical Systems - B, vol. 26, no. 9, pp. 4681-4695, 2021.

[14] S. Ji and G. Li, "A unified approach to nonlocal impulsive differential equations with the measure of noncompactness," Advances in Difference Equations, vol. 2012, no. 1, Article ID $182,2012$.
[15] B. Zhu, B. Han, and L. Liu, "Existence of mild solutions for a class of fractional semilinear integro-differential equation of mixed type," Acta Mathematica Scientia, vol. 39A, pp. 13341341, 2019.

[16] H. Gou and B. Li, "Local and global existence of mild solution to impulsive fractional semilinear integro-differential equation with noncompact semigroup," Communications in Nonlinear Science and Numerical Simulation, vol. 42, pp. 204-214, 2017.

[17] L. Liu, F. Guo, C. Wu, and Y. Wu, "Existence theorems of global solutions for nonlinear Volterra type integral equations in Banach spaces," Journal of Mathematical Analysis and Applications, vol. 309, no. 2, pp. 638-649, 2005.

[18] S. Samko, A. Kilbas, and O. Marichev, Fractional Integrals and Derivatives: Theory and Applications, Gordon and Breach, Amsterdam, 1993.

[19] M. Caputo, "Linear models of dissipation whose $q$ is almost frequency independent-II," Journal of the Royal Astronomical Society, vol. 13, no. 5, pp. 529-539, 1967.

[20] D. Araya and C. Lizama, "Almost automorphic mild solutions to fractional differential equations," Nonlinear Analysis, vol. 69, no. 11, pp. 3692-3705, 2008.

[21] A. Debbouche and D. Baleanu, "Controllability of fractional evolution nonlocal impulsive quasilinear delay integrodifferential systems," Computers and Mathematics with Applications, vol. 62, no. 3, pp. 1442-1450, 2011.

[22] C. Lizama, A. Pereira, and R. Ponce, "On the compactness of fractional resolvent operator functions," Semigroup Forum, vol. 93, no. 2, pp. 363-374, 2016. 